\title{
A NOTE ON GOOD REDUCTION OF SIMPLE ABELIAN VARIETIES ${ }^{1}$
}

\author{
C. ADIMOOLAM
}

\begin{abstract}
In this note it is shown that the reduction of a simple abelian variety of dimension $>2$, defined over an algebraic number field, at any finite good prime need not be simple. We give an example of a two-dimensional simple abelian variety defined over an algebraic number field whose reduction at any finite prime is isogenous either to a product of ordinary elliptic curves or to a product of supersingular elliptic curves.
\end{abstract}

It is well known [7] that an abelian variety $A$ defined over an algebraic number field $K$ has good reduction almost everywhere. In other words, if $O$ is the ring of integers in $K, \Sigma$ the set of all finite primes of $K$, and $S$ a finite subset of $\Sigma$, then for every prime $p$ in $\Sigma-S$, there exists an abelian scheme $X$ over $O_{p}$ such that $X \times{ }_{o_{p}} K \simeq A$. Serre and Tate [6] have also proved that if $A$ has sufficiently many complex multiplications, then it has potentially good reduction everywhere. It is clear that if the reduction $\tilde{A}$ of an abelian variety $A$ is simple, then $A$ itself is simple, because $\operatorname{End}^{0}(A) \subseteq \operatorname{End}^{0}(\tilde{A})$ and the latter is a division algebra over $\mathbf{Q}$. One can ask whether the converse is true. More precisely, if $A$ is simple, then is $\tilde{A}$ also simple? In this note we shall see that this need not be the case.

Notations and terminology. We employ the following notation:

$\mathbf{Z}=$ the ring of rational integers; $\mathbf{Q}=$ the field of rationals;

$\mathbf{Z}_{p}=$ the ring of $p$-adic integers; $\mathbf{Q}_{p}=$ the field of fractions of $\mathbf{Z}_{p}$;

$\overrightarrow{\mathbf{Q}}_{p}=$ the algebraic closure of $\mathbf{Q}_{p} ; \mathbf{C}=$ the field of complex numbers.

The principal reference for abelian varieties is [4]. If $A$ is an abelian variety defined over a field $K$, let $\operatorname{End}^{0}(A)=\operatorname{End}_{K}(A) \otimes \mathbf{Q}$. If the characteristic of $K$ is $p>0$, let $T_{p}(A)$ denote the Barsotti-Tate group ( $p$-divisible group in the terminology of Serre and Tate) associated to $A$. It has height $2 g$, where $g$ is the dimension of $A . T_{p}(A)_{\text {red }}=$ the étale part of $T_{p}(A)$. Let $M$ denote the Dieudonné module of $T_{p}(A)$. If $K$ is a perfect field, then $M$ is a module over $W(K)[F, V]$, where $W(K)$ is the ring of infinite Witt vectors over $K$ and $F, V$

Received by the editors June 22, 1976 and, in revised form, November 29, 1976.

AMS (MOS) subject classifications (1970). Primary 14K05, 14K15; Secondary 14L05.

Key words and phrases. Simple abelian variety, good reduction, p-rank of an abelian variety, Barsotti-Tate group, Dieudonné module, indefinite quaternion algebra, ordinary and supersingular elliptic curves.

'This note is taken from author's Ph. D. thesis, SUNY at Stony Brook, May 1975. The author thanks his thesis advisor Professor M. Fried for his encouragement and Professor J.-P. Serre for suggesting the example discussed in Corollary 1. 
are indeterminates such that (i) $F V=V F=p$ and (ii) $F \alpha=\alpha^{\sigma} F, \alpha V=V \alpha^{\sigma}$ for $\alpha \in W(K)$; here $\sigma$ is the unique automorphism of $W(K)$ inducing the map $x \rightarrow x^{p}$ in $K$. The module $M$ is free of rank $2 g$ over $W(K)$. For details on Dieudonné modules, see [5]. The integer $p(A)$ will denote the $p$-rank of an abelian variety $A$.

THEOREM. Let $A$ be an abelian variety of dimension $\geqslant 2$, defined over a field $k$ of positive characteristic $p$. Let $p(A)=1$. Then $\operatorname{End}^{0}(A)$ will never contain a simple subalgebra which is not a field.

Proof. Suppose that $\operatorname{End}^{0}(A)$ contains a simple subalgebra $L$ such that $1_{A}=1_{L}$. Let $C$ be the center of $L$. Then from the general theory of simple algebras, we have $[L: C]=d^{2}$ and $[C: Q]=e$ for some natural numbers $d$ and $e$. Let $\rho$ be the representation of $L \otimes \mathbf{Q}_{p}$ on the extended Dieudonné module $M \otimes \mathbf{Q}_{p}$ of $T_{p}(A)$. This representation is induced from the representation of $\operatorname{End}^{0}(A)$. It has degree $2 g$ over $W(k) \otimes \mathbf{Q}_{p}$. The representation $\rho$ splits into three parts corresponding to the splitting of $M$ into $M^{\text {ét,ét }} \oplus M^{\text {ét,loc }}$ $\oplus M^{\text {loc,loc }}$, where $M^{\text {étét }}$ is the étale part of $M$ whose Cartier dual is also étale, etc. Write $\rho=\rho_{1} \oplus \rho_{2} \oplus \rho_{3}$. The first representation $\rho_{1}$ is the representation over $\mathbf{Q}_{p}$ of $L \otimes \mathbf{Q}_{p}$ on the $p$-adic Tate module $T_{p}(A)_{\text {red }} \otimes \mathbf{Q}_{p}$. Since $p(A)=1$, $T_{p}(A) \otimes \mathbf{Q}_{p}$ is isomorphic to $\mathbf{Q}_{p}$. Decompose $L \otimes \overline{\mathbf{Q}}_{p}$ into a product $\Pi_{1}^{e} M_{d}\left(\overline{\mathbf{Q}}_{p}\right)$, where $M_{d}\left(\overline{\mathbf{Q}}_{p}\right)$ is the algebra of matrices of degree $d$ over $\overline{\mathbf{Q}}_{p}$. Since the identity of $L$ is represented by the identity matrix, the representation $\rho_{1}$ does not contain the zero representation. Consequently $d=1$; that is $L$ is a field. Q.E.D.

Corollary 1. Let $M_{2}$ be the one-dimensional moduli scheme of two-dimensional abelian varieties defined over $\mathbf{C}$ whose endomorphism ring is an order in an indefinite quaternion division algebra $\mathrm{H}$ over $\mathbf{Q}$. Then the fiber at any closed point is a simple abelian variety over an algebraic number field $K$ whose reduction at every finite prime of $K$ is either ordinary or has p-rank 0.

REMARK. The abelian varieties corresponding to closed points as in Corollary 1 are called false elliptic curves by J.-P. Serre for the reason that they behave like elliptic curves when we reduce at a finite prime. Recall that an elliptic curve in positive characteristic is either ordinary or supersingular, i.e. the $p$-rank is either one or zero.

Proof of Corollary 1. As Shimura has observed in [8], $M_{2}$ has a canonical nonsingular model defined over $\mathbf{Q}$ which we again denote by $\boldsymbol{M}_{2}$. Let $A$ be a two-dimensional abelian variety corresponding to any closed point of $M_{2} . A$ is defined over a number field, say, $K$. It has potential good reduction at any finite prime of $K$. For example, see [3]. By extending $K$ to a finite extension if necessary, we can assume that $A$ has good reduction everywhere. For any finite prime $p$ of $K$, let $A^{p}$ be the reduction of $A$ at $p \cdot \tilde{A}^{p}$ is defined over a finite field of characteristic $p$. Since $\operatorname{End}^{0}(A) \subseteq \operatorname{End}^{0}\left(\tilde{A}^{p}\right)$, $\operatorname{End}^{0}\left(A^{p}\right)$ contains an indefinite quaternion division algebra and so by the 
theorem, $\tilde{A}^{p}$ can not have $p$-rank one. Hence either $\tilde{A}^{p}$ is ordinary or it has p-rank 0. Q.E.D.

Corollary 2. Let $A$ be a two-dimensional abelian variety as in Corollary 1. $A$ is simple (even absolutely simple) and it is defined over a number field $K$. Then for any finite $p$ of $K, A^{p}$ is not simple.

Proof. By Corollary 1 , either $\tilde{A}^{p}$ is ordinary or it has $p$-rank zero. The following idea of the proof is due to Deligne-Rapoport [2]. $\theta=\operatorname{End}_{K}(A)$ is an order in the given indefinite quaternion division algebra $H$. We have an embedding of $\theta$ in $\operatorname{End}_{k}\left(\tilde{A}^{p}\right)$ where $k$ is the residue field at the prime $p$. Hence $\theta$ can be considered as a ring of operators of $\tilde{A}^{p}$. Let $u$ be a nontrivial idempotent of $\theta$ (note that such an idempotent exists, since $\theta$ is a nonnilpotent subring of $H$ ). Then $e=u \otimes 1$ is a nontrivial idempotent of $\theta \otimes \mathbf{Z}_{p}$. Let $T_{p}\left(\tilde{A}^{p}\right)$ denote the Barsotti-Tate group of the 2-dimensional abelian variety $\hat{A}^{p}$. It has height 4 . The completion of $\theta \otimes \mathbf{Z}_{p}$ acts on $T_{p}\left(\tilde{A}^{p}\right)$. Write $T_{p}\left(\tilde{A}^{p}\right)=e T_{p}\left(\tilde{A}^{p}\right) \oplus(1-e) T_{p}\left(\tilde{A}^{p}\right)$. Both these components are BarsottiTate subgroups of $T_{p}\left(\tilde{A}^{p}\right)$, each of height 2 . Corresponding to this decomposition, $\tilde{A}^{p}$ can be written up to isogeny as a product of elliptic curves: $\tilde{A}^{p} \sim E \times F$, where $E=$ image of the endomorphism $u$ in $\tilde{A}^{p}$, whose associated Barsotti-Tate group is $e T_{p}\left(\tilde{A}^{p}\right)$ and $F$ is the connected component of the identity of the kernel of $u$. Since $\tilde{A}^{p}$ has $p$-rank either 0 or 2 , both $E$ and $F$ have $p$-rank either 0 or 1 , since $p$-rank is additive and isogeny invariant. This proves that the reduction $\tilde{A}^{p}$ of $A$ is everywhere nonsimple. Indeed, it is isogenous either to a product of supersingular or ordinary elliptic curves. Q.E.D.

I thank the referee for some useful comments.

\section{REFERENCES}

1. C. Adimoolam, Moduli of polarized abelian varieties and complex multiplications, Ph.D. thesis, SUNY at Stony Brook, May 1975.

2. P. Deligne and M. Rapoport, Les schémas de modules de courbes elliptiques, Modular Functions of One Variable, II, Lecture Notes in Math., vol. 349, Springer-Verlag, Berlin and New York, 1972. MR 49 \#2762.

3. Y. Morita, On potential good reduction of abelian varieties, preprint.

4. D. Mumford, Abelian varieties, Oxford University Press, London, 1970. MR 44 \#219.

5. T. Oda, The first de Rham cohomology group and Dieudonné modules, Ann. Sci. Ecole Norm. Sup. (4) 2 (1969), 63-135. MR 39 \#2775.

6. J.-P. Serre and J. Tate, Good reduction of abelian varieties, Ann. of Math. (2) 88 (1968), 492-517. MR 38 \#4488.

7. G. Shimura, Reduction of algebraic varieties with respect to a discrete valuation of the basic field, Amer. J. Math. 77 (1955), 134-176. MR 16, 616.

8. On the zeta functions of algebraic curves uniformized by certain automorphic functions, J. Math. Soc. Japan 13 (1961), 275-331. MR 26 \#84.

Department of Mathematics, University of California, Irvine, California 92717 